\title{
Sensor Technologies and Methods for Geoinformatics and Remote Sensing
}

\author{
Hyung-Sup Jung $\mathbb{D},{ }^{1}$ Saro Lee $\mathbb{D}^{2,3}$ and Lei Zhang $\mathbb{D}^{4}$ \\ ${ }^{1}$ Department of Geoinformatics, University of Seoul, Seoul, Republic of Korea \\ ${ }^{2}$ Korea Institute of Geoscience and Mineral Resources (KIGAM), Daejeon, Republic of Korea \\ ${ }^{3}$ Department of Geophysical Exploration, Korea University of Science and Technology, Daejeon, Republic of Korea \\ ${ }^{4}$ The Department of Land Surveying and Geo-Informatics, Hong Kong Polytechnic University, Hung Hom, Hong Kong
}

Correspondence should be addressed to Hyung-Sup Jung; hsjung@uos.ac.kr

Received 13 March 2018; Accepted 13 March 2018; Published 23 May 2018

Copyright (C) 2018 Hyung-Sup Jung et al. This is an open access article distributed under the Creative Commons Attribution License, which permits unrestricted use, distribution, and reproduction in any medium, provided the original work is properly cited.

\section{Introduction}

The design, performance, and application of sensors for geoinformatics and remote sensing have become more essential to understanding the physical, ecological, hydrological, and environmental characteristics of earth surfaces. In order to address the state-of-the-art sensor technologies and methods in applying geospatial technologies, which include geospatial information sciences (GIS), remote sensing (RS), photogrammetry, and global positioning system (GPS), this special issue of the Journal of Sensors, "Sensor Technologies and Methods for Geoinformatics and Remote Sensing," was successfully planned. The objective of this special issue is to create a multidisciplinary forum of discussion on recent advances in sensor technologies and methods for geoinformatics and remote sensing as well as new applications to biology, ecology, hydrology, engineering, and we here publish many papers detailing novel contributions that are of relevance to these topics.

\section{Sensor Technologies and Methods for Geoinformatics and Remote Sensing}

In total, 33 papers were submitted to this special issue, 18 of which were accepted and published, constituting a 55\% acceptance rate. The papers in this special issue cover various areas related to the design, performance, and application of sensors for geoinformatics and remote sensing, which are typical tools used by geoinformation researchers. The published papers addressed the followings:

(i) Forest height estimation, rock identification, mining subsidence, $2 \mathrm{D}$ ground deformation, snow cover detection, change detection, evapotranspiration retrieval, and pyroclastic flow mapping from the remote-sensed imagery

(ii) Orthorectification of optical images and ionospheric correction of SAR interferometry

(iii) Development of omnidirectional image-based data model, tile-based rendering method, aeromagnetic compensation algorithm, and micromotion feature extraction method for the geoinformation

(iv) Two-dimensional dead reckoning based on vehicle dynamic sensors during GNSS outages

(v) Landslide susceptibility assessment and land-use change simulation using geospatial information system

(vi) Research trend analysis of geospatial information in South Korea using text-mining technology 


\section{Acknowledgments}

We would like to thank all the authors for their valuable contributions in this special issue as well as all the reviewers who are all experts on the theme. This special issue was conducted by the Korea Meteorological Administration Research and Development Program under Grant no. KMI2017-9060. This special issue was conducted by the Basic Research Project of the Korea Institute of Geoscience and Mineral Resources (KIGAM) funded by the Ministry of Science and ICT.

Hyung-Sup Jung Saro Lee Lei Zhang 


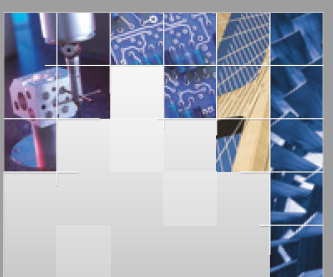

\section{Enfincering}
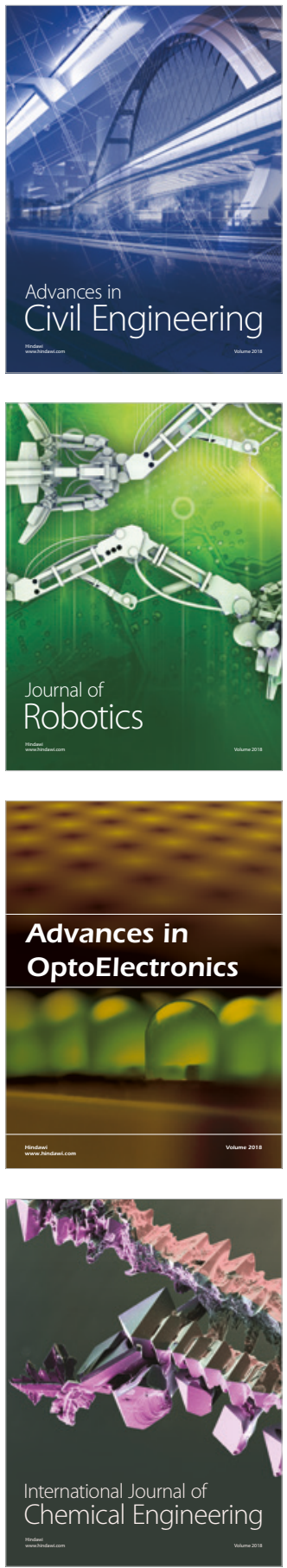

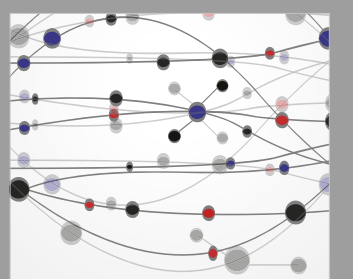

\section{Rotating \\ Machinery}

The Scientific World Journal

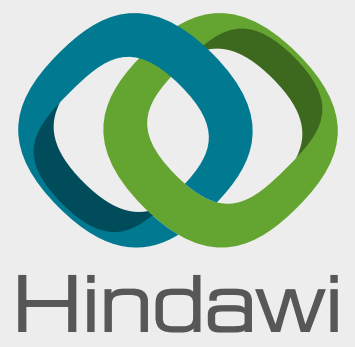

Submit your manuscripts at

www.hindawi.com
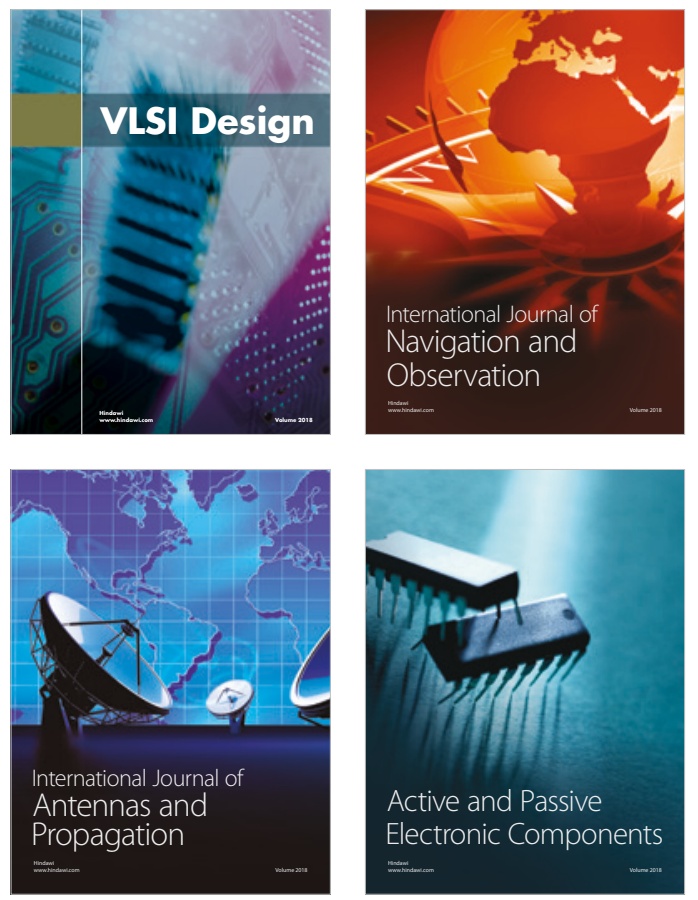
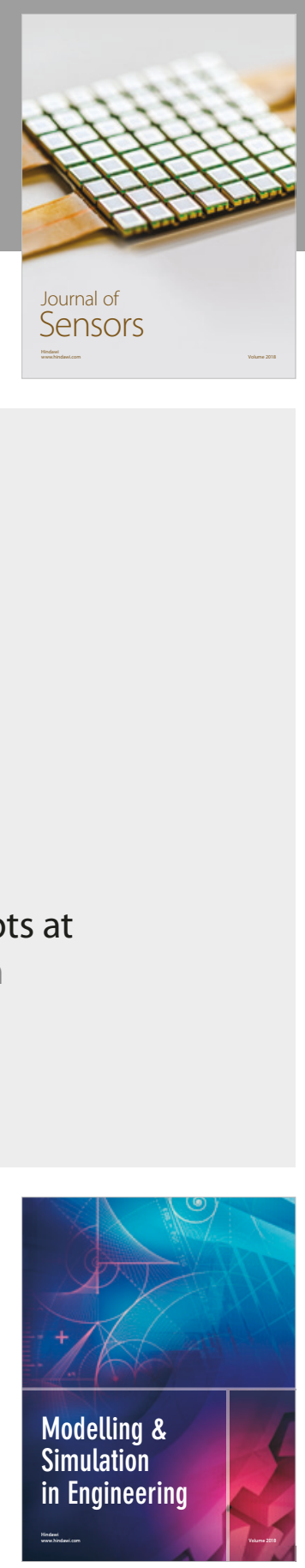

\section{Advances \\ Multimedia}
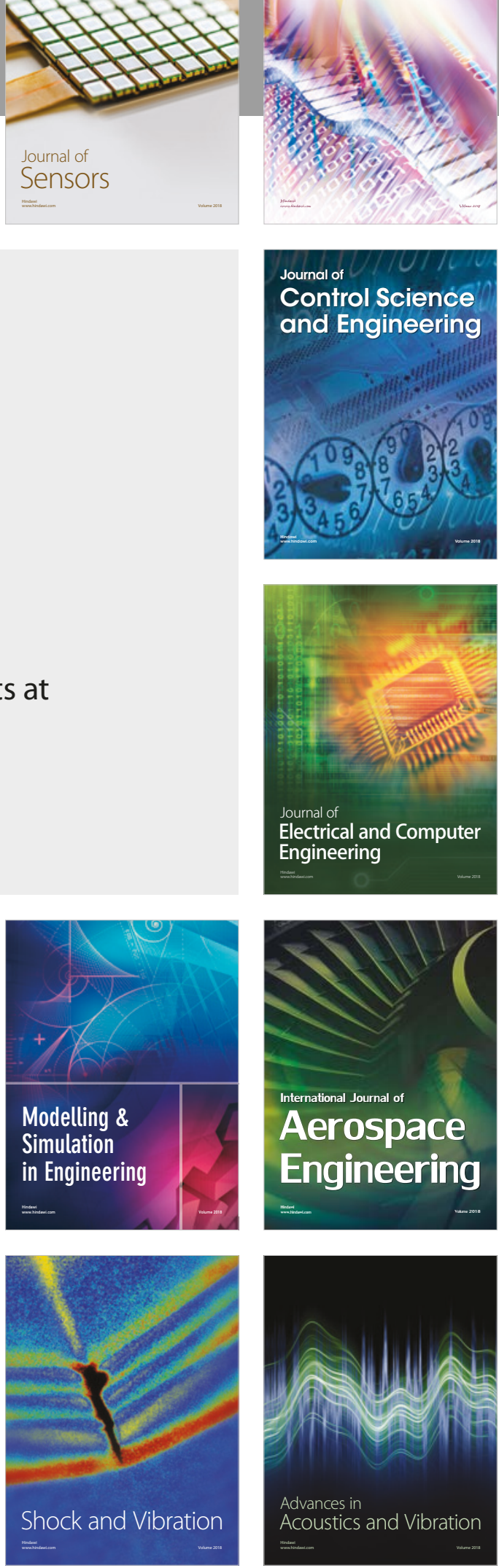